\title{
TRANSFERÊNCIA DE OXIGÊNIO EM BIORREATOR AIRLIFT PRESSURIZADO
}

\author{
G. CAMPANI ${ }^{1}$, A. C. L. HORTA ${ }^{1}$, M. P. A. RIBEIRO ${ }^{1}$, R. C. GIORDANO ${ }^{1}$, A. C. BADINO ${ }^{1}$, T. \\ C. ZANGIROLAMI ${ }^{1}$
}

${ }^{1}$ Programa de Pós-Graduação em Engenharia Química, Universidade Federal de São Carlos E-mail para contato: gilson.campani@yahoo.com

\begin{abstract}
RESUMO - O biorreator airlift apresenta vantagens frente aos reatores convencionais (tanque agitado e aerado), como menor complexidade de construção, baixo risco de contaminação e maior eficiência energética na transferência de oxigênio. Entretanto, a menor transferência de oxigênio no airlift, em relação à alcançada em reator convencional, justifica a pressurização do tanque a fim de maximizar esse transporte de massa. Neste âmbito, o objetivo deste trabalho foi avaliar a influência da pressão sobre a retenção gasosa $(\varepsilon)$ e o coeficiente volumétrico de transferência de oxigênio $\left(\mathrm{k}_{\mathrm{L}} \mathrm{a}\right)$ em biorreator airlift. Os experimentos foram conduzidos em airlift de cilindros concêntricos de $5 \mathrm{~L}$ operado com água e meio de cultura complexo. Mostrou-se que uma pressão de até 4 bar não afeta $\varepsilon$ nem $\mathrm{k}_{\mathrm{L}} \mathrm{a}$. A compreensão do efeito da pressão do sistema sobre a transferência de oxigênio no biorreator airlift contribui grandemente para a área de desenvolvimento de reatores não convencionais.
\end{abstract}

\section{INTRODUÇÃO}

Dentre os diversos reatores utilizados em bioprocessos, o biorreator pneumático airlift é um exemplo de reator não convencional que apresenta características instrumentais e de operação bastante interessantes. Basicamente, esse reator é composto por duas regiões interligadas no topo e na base do equipamento: a região de subida, onde o gás é aspergido, e a região de descida, que canaliza o meio reacional de volta para a base do reator. Com isso, há a mistura e a circulação do meio reacional dentro do biorreator. De forma geral, a menor complexidade de construção, o menor risco de contaminação decorrente da inexistência do selo mecânico (presente no tanque mecanicamente agitado) e a eficiente dispersão gás-líquido realizada pelo próprio gás de entrada com baixo consumo de energia (Onken e Weiland, 1983) fazem do biorreator airlift uma alternativa interessante frente aos reatores convencionais (tipo tanque agitado e aerado).

A ausência de agitação mecânica no biorreator airlift acarreta em menor transferência de oxigênio. $\mathrm{O}$ transporte de massa da fase gasosa para a fase líquida, no entanto, pode ser elevado através da manipulação de variáveis não convencionais, tal como a sobrepressão do reator. Letzel et al. (1999) relataram um aumento de retenção gasosa $(\varepsilon)$ e do coeficiente volumétrico de transferência de oxigênio $\left(\mathrm{k}_{\mathrm{L}} \mathrm{a}\right)$ decorrente da sobrepressão em biorreator tipo coluna de bolhas. $\mathrm{O}$ mesmo efeito foi também observado por Letzel e Stankiewicz (1999) para biorreator airlift de circulação externa.

De acordo com a teoria de instabilidade hidrodinâmica de Kelvin-Helmoltz, a elevação da pressão em um sistema gás-líquido desestabiliza as bolhas de gás com maiores diâmetros (1,5 - 
5,0 cm), diminuindo o seu tamanho médio (Kitscha e Kocamustafaogullari, 1989; Wilkinson e Dierendonck, 1990). Com isso, há um aumento da área interfacial gás-líquido específica de transferência de massa e uma diminuição na velocidade terminal de ascensão das bolhas de gás na coluna de líquido (Talaia, 2008), ambos afetando positivamente $\varepsilon$ e $\mathrm{k}_{\mathrm{L}} \mathrm{a}$.

Embora a influência da pressão sobre a hidrodinâmica e a transferência de massa esteja bem estabelecida para os reatores coluna de bolhas e airlift de circulação externa, inexistem estudos na literatura envolvendo o biorreator airlift de circulação interna. Portanto, nesse contexto, este trabalho objetivou investigar a influência da pressão do sistema sobre $\varepsilon, \mathrm{k}_{\mathrm{L}} \mathrm{a}$ e sobre a transferência de oxigênio em biorreator airlift de cilindros concêntricos em escala de bancada (5 L).

\section{MATERIAIS E MÉTODOS}

\subsection{Fluidos}

Para a realização dos experimentos no biorreator airlift, foram empregadas as seguintes fases líquidas: água destilada e meio de cultura de autoindução (Studier, 2005).

A composição do meio de cultura é: glicose $(10 \mathrm{~g} / \mathrm{L})$, glicerol $(60 \mathrm{~g} / \mathrm{L})$, lactose $(20 \mathrm{~g} / \mathrm{L})$, phytone $(10 \mathrm{~g} / \mathrm{L})$, extrato de levedura $(5 \mathrm{~g} / \mathrm{L}), \mathrm{Na}_{2} \mathrm{HPO}_{4}(9 \mathrm{~g} / \mathrm{L}), \mathrm{KH}_{2} \mathrm{PO}_{4}(3,4 \mathrm{~g} / \mathrm{L}), \mathrm{NH}_{4} \mathrm{Cl}(2,7$ $\mathrm{g} / \mathrm{L}), \mathrm{Na}_{2} \mathrm{SO}_{4}(0,7 \mathrm{~g} / \mathrm{L})$, citrato de ferro (100,8 mg/L), $\mathrm{CoCl}_{2} .6 \mathrm{H}_{2} \mathrm{O}(2,5 \mathrm{mg} / \mathrm{L}), \mathrm{MnCl}_{2} .4 \mathrm{H}_{2} \mathrm{O}(15$ $\mathrm{mg} / \mathrm{L}), \quad \mathrm{CuCl}_{2} \cdot 2 \mathrm{H}_{2} \mathrm{O} \quad(1,5 \mathrm{mg} / \mathrm{L}), \quad \mathrm{H}_{3} \mathrm{BO}_{3} \quad(3 \mathrm{mg} / \mathrm{L}), \quad \mathrm{Na}_{2} \mathrm{MoO}_{4} \cdot 2 \mathrm{H}_{2} \mathrm{O} \quad(2,1 \mathrm{mg} / \mathrm{L})$, $\mathrm{Zn}\left(\mathrm{CH}_{3} \mathrm{COOH}\right)_{2} \cdot \mathrm{H}_{2} \mathrm{O}(33,8 \mathrm{mg} / \mathrm{L})$, EDTA $(14,1 \mathrm{mg} / \mathrm{L}), \mathrm{MgSO}_{4} .7 \mathrm{H}_{2} \mathrm{O}(0,5 \mathrm{~g} / \mathrm{L})$, tiamina $(45$ $\mathrm{mg} / \mathrm{L})$, canamicina $(100 \mathrm{mg} / \mathrm{L})$, polipropileno glicol $(0,03 \% \mathrm{v} / \mathrm{v})$ e $\mathrm{pH}$ de 6,7 . A água destilada e o meio de cultivo são fluidos newtonianos, com viscosidades dinâmicas de $0,8.10^{-3}$ Pa.s e $1,2.10^{-3}$ Pa.s, respectivamente, a $30^{\circ} \mathrm{C}$. A viscosidade do meio de cultura foi aferida através de reômetro digital de cilindros concêntricos (Brookfield Engineering Laboratories, model LV-DVIII+).

O gás aspergido no reator foi composto por misturas de nitrogênio e ar em proporções nas quais a pressão parcial de oxigênio se limitasse a 0,21 bar, mesmo nas condições de pressurização do biorreator. Isso foi necessário uma vez que o sensor amperométrico de oxigênio dissolvido não é capaz de aferir concentrações de oxigênio dissolvido superiores à de saturação com ar à pressão atmosférica (aproximadamente $7 \mathrm{mg} / \mathrm{L}$ ).

\subsection{Equipamento}

Os experimentos foram conduzidos em biorreator penumático airlift de cilindros concêntricos com $5 \mathrm{~L}$ de volume útil. $\mathrm{O}$ reator foi adaptado do projeto originalmente desenvolvido por Badino et al. (2004), substituindo o tubo superior por um confeccionado em aço inox (316L) contendo uma janela em vidro para observação do interior do equipamento. $O$ aparato experimental contou com controle automático de pressão e temperatura desenvolvido em LabVIEW (National Instruments®, versão 8). A válvula atuadora (SMC, modelo VY1100) e o sensor de pressão (SMC, modelo ISE30A-01-F) foram instalados na linha de saída de gás. Os dados online foram adquiridos por meio de conversor A/D e D/A, compact FieldPoint (National Instruments, modelo cFP2020). A concentração de oxigênio dissolvido foi determinada através de sensor amperométrico (Mettler Toledo, modelo InPro 6830) munido com membrana de silicone (modelo S96). Um esquema do equipamento é representado na Figura 1. 


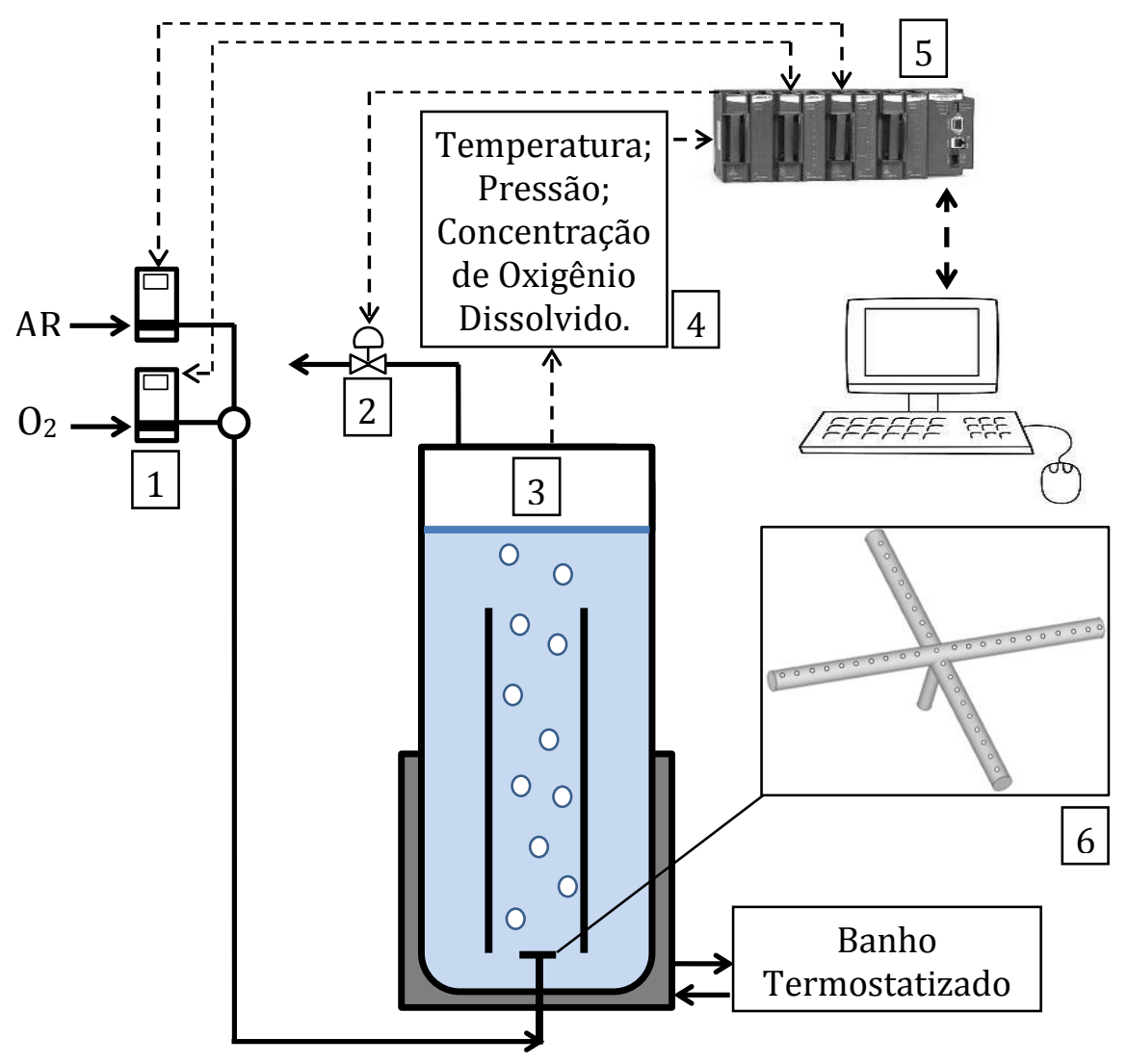

Figura 1 - Aparato experimental utilizado. (1) fluxômetros mássicos de gás (AALBORG®, modelo GFC); (2) válvula atuadora da linha de saída de gás; (3) biorreator airlift; (4) sensores de temperatura, concentração de oxigênio dissolvido e pressão; (5) compact FieldPoint; (6) aspersor de gás tipo "cruzeta".

\subsection{Medidas da Retenção Gasosa e do Coeficiente Volumétrico de Transferência de Oxigênio}

A retenção gasosa global $(\varepsilon)$ foi determinada pelo método de expansão volumétrica (Chisti, 1989), onde as alturas do líquido aerado $\left(\mathrm{h}_{\mathrm{D}}\right)$ e não aerado $\left(\mathrm{h}_{\mathrm{L}}\right)$ dentro do biorreator foram medidas. A partir desses valores, determinou-se $\varepsilon$ através da Equação 1.

$$
\varepsilon=\frac{h_{D}-h_{L}}{h_{D}}
$$

O coeficiente volumétrico de transferência de oxigênio $\left(\mathrm{k}_{\mathrm{L}} \mathrm{a}\right)$ foi avaliado pelo método dinâmico por degrau de pressão (Linek et al., 1989). Nesse método, a pressão do sistema foi subitamente elevada em aproximadamente $15 \%$. Com isso, a concentração de saturação de oxigênio dissolvido foi aumentada de um valor inicial $\left(\mathrm{C}_{\mathrm{e} 0}\right)$ para outro aproximadamente $15 \%$ superior $\left(\mathrm{C}_{\mathrm{eS}}\right)$. Através de regressão não linear pelo método dos mínimos quadrados (Marquardt, 1963), o valor de $\mathrm{k}_{\mathrm{L}}$ a foi obtido ajustando o modelo matemático (Equação 2) aos dados da concentração de oxigênio dissolvido medidos pelo sensor $\left(\mathrm{C}_{\mathrm{e}}\right)$ em função do tempo após o aumento abrupto da pressão. A Equação 2 é proveniente do balanço de massa de oxigênio e no modelo de primeira ordem para a resposta do sensor de oxigênio dissolvido. 


$$
C_{e}=C_{e 0} \cdot e^{-k_{e} \cdot\left(t-t_{0}\right)}+C_{e S} \cdot\left(1-e^{-k_{e} \cdot\left(t-t_{0}\right)}\right)+\frac{k_{e \cdot(}\left(C_{e S}-C_{e 0}\right)}{k_{e}-k_{L} a} \cdot\left(e^{-k_{e} \cdot\left(t-t_{0}\right)}-e^{-k_{L} a \cdot\left(t-t_{0}\right)}\right)
$$

onde $\mathrm{C}_{\mathrm{e}}=\mathrm{C}_{\mathrm{e} 0}$ no instante inicial $\left(\mathrm{t}=\mathrm{t}_{0}\right)$ e $\mathrm{k}_{\mathrm{e}}$ é a constante de atraso do sensor estimada juntamente com o $\mathrm{k}_{\mathrm{L}}$ a pelo método dos mínimos quadrados (Marquardt, 1963).

Para os experimentos conduzidos às pressões de 2, 3 e 4 bar, a vazão volumétrica de gás foi mantida constante dentro do reator através da elevação da vazão mássica do gás de acordo com a lei dos gases ideais (Equação 3).

$$
Q=\frac{\dot{m}}{P} \cdot \frac{R \cdot T}{M M}
$$

onde $\mathrm{P}$ é a pressão absoluta, $\mathrm{Q}$ a vazão volumétrica de gás, $\dot{m}$ a vazão mássica de gás, T a temperatura absoluta, MM a massa molar do gás e R a constante universal dos gases ideais.

Finalmente, para avaliar estatisticamente a influência da pressão sobre o $\mathrm{k}_{\mathrm{L}} \mathrm{a}$, ajustou-se uma correlação empírica generalizada, representada na Equação 4, aos dados experimentais obtidos para as diferentes condições de P e Q empregadas.

$$
k_{L} a=b \cdot Q^{c} \cdot P^{d}
$$

onde b, c e d são constantes estimadas através de regressão não linear por mínimos quadrados (Marquardt, 1963). As constantes são adimensionais, exceto $\mathrm{b}\left[\mathrm{s}^{\mathrm{c}-1} \mathrm{~m}^{-3 \mathrm{c}} \mathrm{Pa}^{-\mathrm{d}}\right]$.

\subsection{Estimativa da Velocidade de Transferência de Oxigênio}

De acordo com teoria das duas películas estagnadas, a velocidade volumétrica de transferência de oxigênio $\left(N_{\mathrm{O}_{2}}\right)$ da fase gasosa para a líquida pode ser descrita como o produto de uma força motriz $\left(\mathrm{C}^{*}-\mathrm{C}\right)$ pelo $\mathrm{k}_{\mathrm{L}} \mathrm{a}$ (Equação 5) (Garcia-Ochoa e Gomez, 2009).

$$
N_{O_{2}}=k_{L} a \cdot\left(C^{*}-C\right)=k_{L} a \cdot\left[\left(\frac{y_{O_{2}}}{H_{O_{2} / \text { líquido }}} \cdot P\right)-C\right]
$$

onde $C^{*}$ é a concentração de oxigênio na fase líquida em equilíbrio com a sua pressão parcial na fase gasosa, $\mathrm{C}$ a concentração de oxigênio no seio do líquido, $\mathrm{H}_{\mathrm{O} / \text { /líquido }}$ a constante da Lei de Henry para o sistema oxigênio-líquido, yo2 a fração molar de oxigênio no gás da linha de entrada e P a pressão absoluta do gás.

\section{RESULTADOS E DISCUSSÃO}

A partir das Equações 1 e 2, foram estimados os valores de $\varepsilon$ e de $\mathrm{k}_{\mathrm{L}} \mathrm{a}$ utilizando os dados experimentais obtidos para vazões específicas de gás no biorreator airlift de 2, 3 e 4 vvm (V/V.min) e de pressão interna de 1, 2 e 3 bar. Para a pressão de 4 bar, as vazões específicas empregadas foram de 2 e $3 \mathrm{vvm}$. Não foi possível obter os valores de $\varepsilon$ e de $\mathrm{k}_{\mathrm{L}} \mathrm{a}$ para a vazão de gás de 4 vvm e pressão de 4 bar, uma vez que seria necessário empregar uma vazão mássica superior ao limite de operação dos fluxômetros disponíveis.

Os resultados obtidos para $\mathrm{k}_{\mathrm{L}} \mathrm{a}$ e $\varepsilon$, empregando água e meio de cultivo de autoindução, são apresentados nas Figuras 2 e 3 , respectivamente. Os dados de $\mathrm{k}_{\mathrm{L}} \mathrm{a}$ foram então 
correlacionados com a vazão volumétrica de gás (Q) e com a pressão absoluta interna do biorreator $(\mathrm{P})$, de acordo com a Equação 4. As correlações obtidas para $\mathrm{k}_{\mathrm{L}} \mathrm{a}$ em função de $\mathrm{P}$ e Q para o biorreator airlift operado com água e com meio de cultivo são representadas nas Equações 6 e 7, respectivamente.

$$
\begin{array}{ll}
k_{L} a=105 \cdot Q^{(0,98 \pm 0,09)} \cdot P^{(0,04 \pm 0,04)} & \left(R^{2}=0,93\right) \\
k_{L} a=338 \cdot Q^{(0,9 \pm 0,1)} \cdot P^{(-0,15 \pm 0,06)} & \left(R^{2}=0,89\right)
\end{array}
$$

onde as unidades são: $\mathrm{k}_{\mathrm{L}} \mathrm{a}\left[\mathrm{s}^{-1}\right], \mathrm{P}[\mathrm{Pa}]$ e $\mathrm{Q}\left[\mathrm{m}^{3} / \mathrm{s}\right]$.

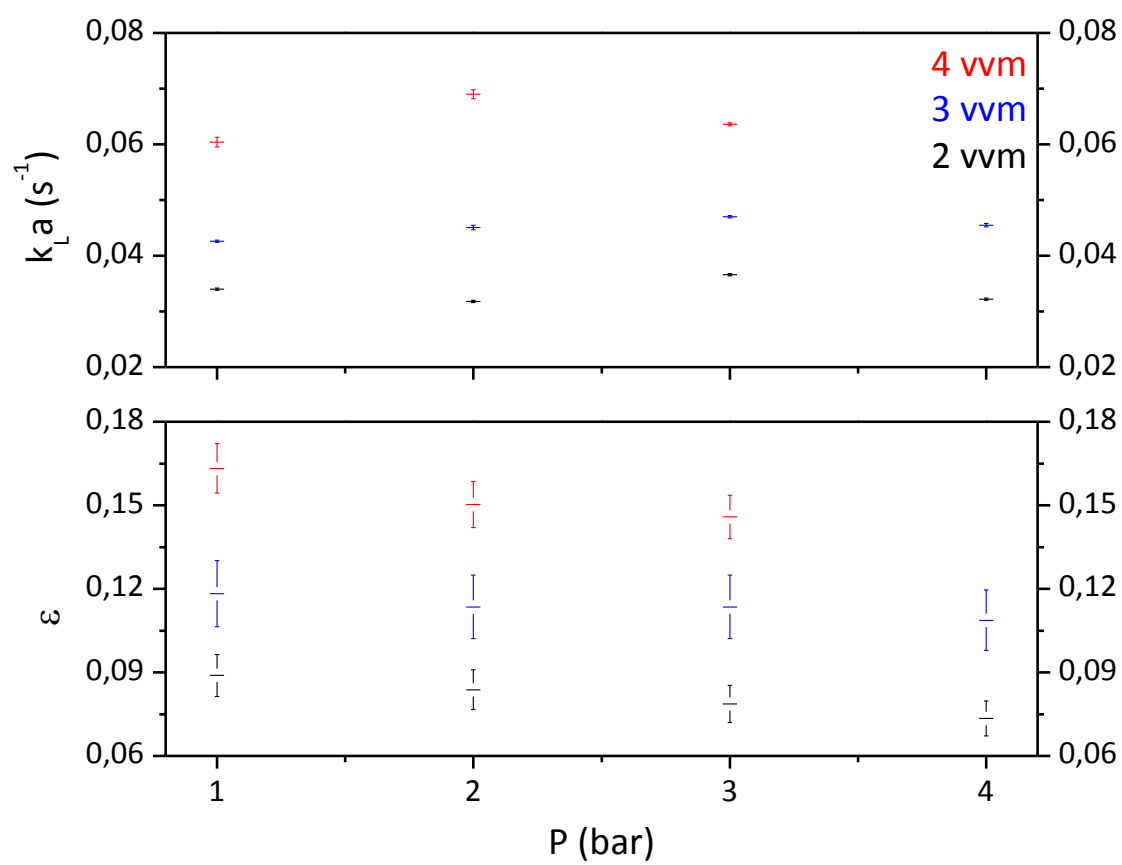

Figura 2 - Medidas em triplicata de $\mathrm{k}_{\mathrm{L}}$ a e $\varepsilon$ em função de $\mathrm{P}$ e $\mathrm{Q}$ em biorreator airlift (5 L) operado com água destilada. As barras de desvio se referem ao desvio padrão das medidas de $\varepsilon$ e ao erro padrão das estimativas de $\mathrm{k}_{\mathrm{L}} \mathrm{a}$.

Analisando as correlações obtidas (Equações 6 e 7), o valor da constante "c" indica que o aumento da vazão de gás atua positivamente no $\mathrm{k}_{\mathrm{L}} \mathrm{a}$, devido ao aumento de $\varepsilon$, conforme já relatado em estudo realizado por Cerri (2009). Já o valor pouco significativo da constante "d", mostra que a pressão interna do biorreator airlift praticamente não afeta o $\mathrm{k}_{\mathrm{L}} \mathrm{a}$. Na Equação 6 (airlift operado com água), o expoente de $\mathrm{P}$ é estatisticamente insignificante, uma vez que o seu valor médio é igual ao seu respectivo erro padrão, indicando a irrelevância do efeito da pressão sobre o $\mathrm{k}_{\mathrm{L}} \mathrm{a}$. Quanto à Equação 7 (airlift operado com meio de cultivo), pode-se afirmar, com base na distribuição estatística de Student, que, com 97\% de confiança, a média do expoente de $\mathrm{P}$ está contida no intervalo $[-0,01 ; 0,31]$. Logo, como esse intervalo contém o valor nulo, se constata novamente a insignificância estatística do expoente de $\mathrm{P}$ e, consequentemente, da influência da pressão sobre o $\mathrm{k}_{\mathrm{L}} \mathrm{a}$. Os menores valores de $\mathrm{k}_{\mathrm{L}} \mathrm{a}$ e $\varepsilon$ obtidos para o meio de cultivo, em comparação aos determinados em água, devem-se à 
presença de polipropileno glicol no meio de cultivo, que aumenta a tensão interfacial gáslíquido e, consequentemente, a coalescência das bolhas de gás.

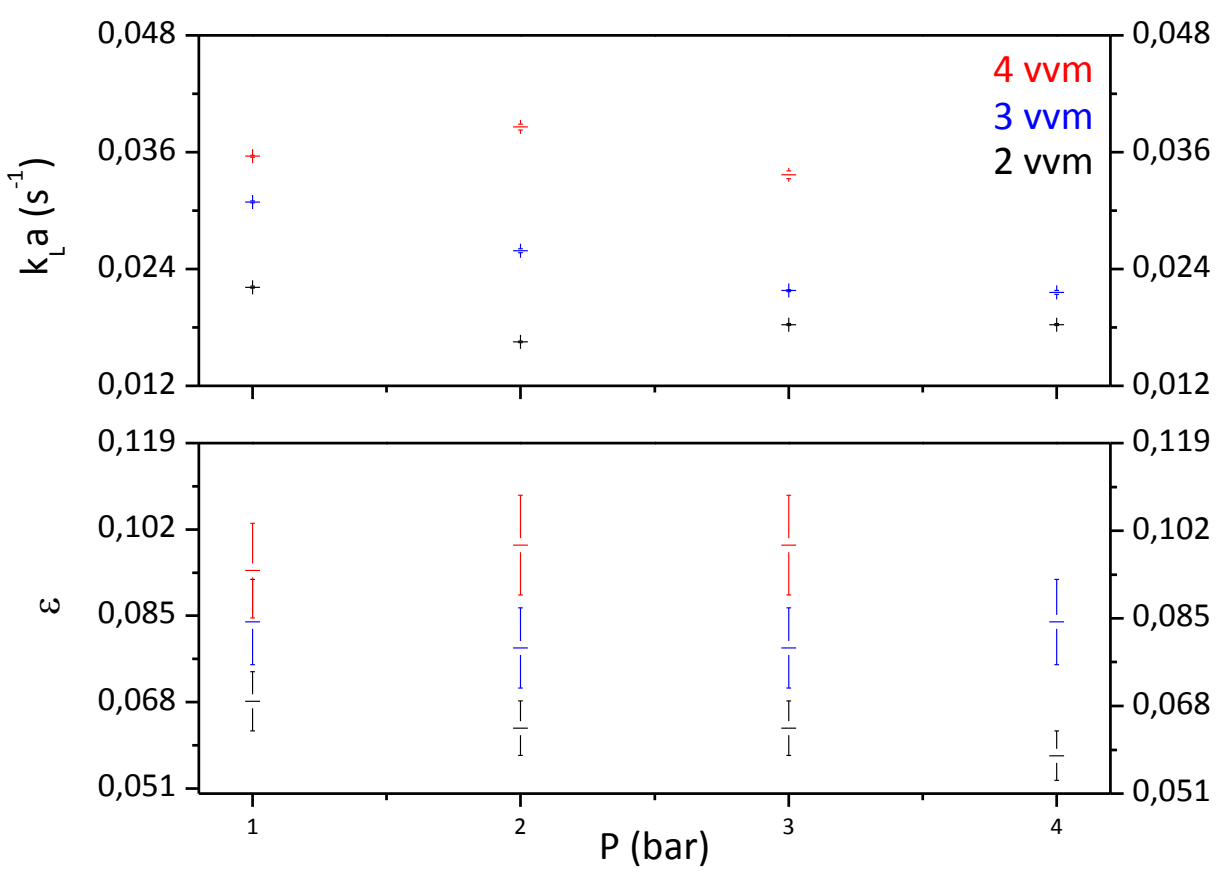

Figura 3 - Medidas em triplicata de $\mathrm{k}_{\mathrm{L}}$ a e $\varepsilon$ em função de $\mathrm{P}$ e $\mathrm{Q}$ em biorreator airlift ( $5 \mathrm{~L}$ ) operado com meio de cultivo. As barras de desvio se referem ao desvio padrão das medidas de $\varepsilon$ e ao erro padrão das estimativas de $\mathrm{k}_{\mathrm{L}} \mathrm{a}$.

$\mathrm{O}$ fato dos parâmetros $\varepsilon$ e $\mathrm{k}_{\mathrm{L}}$ a não serem afetados pela sobrepressão no biorreator airlift de cilindros concêntricos contraria os resultados obtidos em reator tipo coluna de bolhas (Letzel et al., 1999) e em airlift de circulação externa (Letzel e Stankiewics, 1999), onde $\varepsilon$ e $\mathrm{k}_{\mathrm{L}} \mathrm{a}$ aumentaram com a elevação da pressão interna do biorreator.

A elevação da densidade do gás decorrente da pressurização intensifica a fragmentação das bolhas com maiores diâmetros, o que aumenta $\varepsilon$ e $\mathrm{k}_{\mathrm{L}} \mathrm{a}$. Entretanto, esse efeito é considerável apenas para sistemas com maiores velocidades relativas entre as fases gasosa e líquida (Wilkinson e Dierendonck, 1990), como as alcançadas, por exemplo, em reatores tipo coluna de bolhas, próximas de $1,3 \mathrm{~m} / \mathrm{s}$ (Letzel et al., 1999). Isso fica evidenciado no trabalho de Letzel e Stankiewics (1999), no qual é relatado um menor aumento de $\varepsilon$ e $\mathrm{k}_{\mathrm{L}} \mathrm{a}$ com a sobrepressão em airlift de circulação externa, onde a velocidade relativa interfacial gáslíquido $\left(\mathrm{U}_{\mathrm{r}}\right)$ é menor (da ordem de $0,4 \mathrm{~m} / \mathrm{s}$ ), em relação ao aumento observado em reator tipo coluna de bolhas. Logo, possivelmente, devido ao baixo $\mathrm{U}_{\mathrm{r}}$ alcançado no biorreator airlift de circulação interna (por volta de $0,17 \mathrm{~m} / \mathrm{s}$ ), o efeito da sobrepressão sobre a redução do diâmetro médio das bolhas e, consequentemente, sobre $\varepsilon$ e $\mathrm{k}_{\mathrm{L}} \mathrm{a}$ é desprezível nesse reator. A estimativa de $U_{\mathrm{r}}$ para o biorreator airlift de circulação interna foi feita através da Equação 8 (Jones, 1985) utilizando os seguintes dados para o reator airlift de $5 \mathrm{~L}$ operado com água: velocidade superficial do gás na região de subida $\left(\mathrm{U}_{\mathrm{GS}}\right)$ de $0,079 \mathrm{~m} / \mathrm{s}(\mathrm{Q}=20 \mathrm{~L} / \mathrm{min}), \varepsilon=0,16$ e velocidade média de circulação do líquido $\left(\mathrm{U}_{\mathrm{L}}\right)$ de $0,27 \mathrm{~m} / \mathrm{s}$, obtida por Cerri (2009) para semelhante condição de operação no mesmo reator. Utilizando ainda a Equação 8, os valores 
de $U_{\mathrm{r}}$ apresentados para o reator tipo coluna de bolhas e para o reator airlift de circulação externa foram estimados com base nos dados de $\mathrm{U}_{\mathrm{GS}}, \mathrm{U}_{\mathrm{L}}$ e $\varepsilon$ apresentados por Letzel et al. (1999) e Letzel e Stankiewics (1999), respectivamente.

$$
U_{r}=\frac{U_{G S}}{\varepsilon}-U_{L}
$$

Entretanto, mesmo os parâmetros $\varepsilon$ e $\mathrm{k}_{\mathrm{L}}$ a sendo insensíveis à pressão, a mesma pode elevar proporcionalmente $N_{O_{2}}$, como se verifica através da Figura 4, construída a partir da Equação 5 assumindo $\mathrm{C}$ igual a $0,071 \mathrm{~mol} / \mathrm{m}^{3}$ (30\% da saturação em água pura à pressão atmosférica e $30^{\circ} \mathrm{C}$ ). Para a realização dessa estimativa, foram utilizados valores de $\mathrm{k}_{\mathrm{L}} \mathrm{a}$ obtidos no biorreator airlift operado com água destilada e vazão volumétrica de ar de 3 vvm (Figura 3).

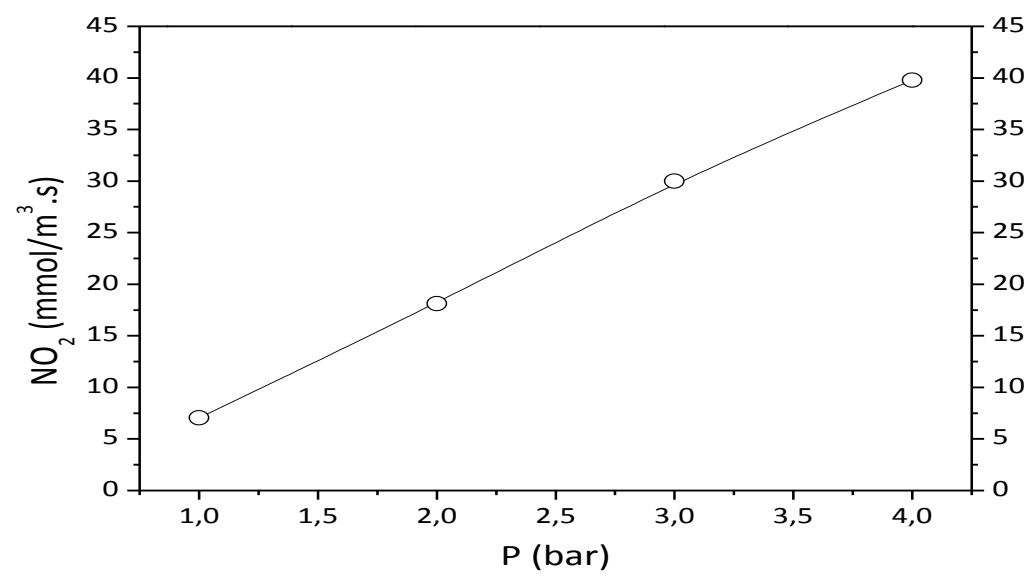

Figura $4-\boldsymbol{N}_{\boldsymbol{O}_{2}}$ predito em função da pressão absoluta (P) em biorreator airlift operado com água destilada e vazão volumétrica de ar de 3 vvm.

\section{CONCLUSÕES}

A caracterização da transferência de oxigênio em biorreator airlift de cilindros concêntricos mostrou que $\varepsilon$ e $\mathrm{k}_{\mathrm{L}}$ a praticamente não são afetados pela pressão (de até 4 bar), independentemente do fluido empregado (água destilada ou meio de cultivo acrescido de antiespumante, com maior tensão interfacial gás-líquido), contrariando os resultados relatados na literatura para reator pneumático tipo coluna de bolhas e airlift de circulação externa, onde $\varepsilon$ e $\mathrm{k}_{\mathrm{L}} \mathrm{a}$ aumentam com a pressão. Com base na teoria de instabilidade hidrodinâmica de Kelvin-Helmoltz e nos resultados adquiridos através do presente trabalho, confirmou-se que $\mathrm{U}_{\mathrm{r}}$ tem papel fundamental nesse fenômeno (quanto menor $\mathrm{U}_{\mathrm{r}}$ menos relevante é o efeito da pressão sobre $\varepsilon$ e $\mathrm{k}_{\mathrm{L}} \mathrm{a}$ em reatores pneumáticos). Entretanto, constatou-se a partir do modelo que descreve a transferência de oxigênio no sistema que a pressão afeta positivamente $N_{O_{2}}$, mesmo com a invariância de $\varepsilon$ e $\mathrm{k}_{\mathrm{L}}$ a devido à pressurização.

Finalmente, convém destacar que a compreensão do efeito da pressão do sistema sobre o transporte de oxigênio é essencial no desenvolvimento de reatores convencionais e não convencionais com maiores capacidades de transferência de oxigênio. 


\section{AGRADECIMENTOS}

Beneficiário de auxílio financeiro da CAPES (Brasil) e da FAPESP (Brasil).

\section{REFERÊNCIAS}

BADINO, A. C.; HOKKA, C. O.; CERRI, M. O. Biorreator pneumático de circulação interna e uso do mesmo. BR n. PI 0404703-6, 2004.

CERRI, M. O. Hidrodinâmica e transferência de oxigênio em três biorreatores airlift de circulação interna geometricamente semelhantes. 2009. 157 f. Tese (Doutorado em Engenharia Química) - Departamento de Engenharia Química, Universidade Federal de São Carlos, São Carlos, 2009.

CHISTI, M. Y. Airlift bioreactors. 1. ed. New York: Elsevier Applied Science, 1989. 345 p.

GARCIA-OCHOA, F.; GOMEZ, E. Bioreactor scale-up and oxygen transfer rate in microbial process: an overview. Biotechnology Advances, v. 27, p. 153-176, 2009.

JONES, A. G. Liquid circulation in a draft-tube bubble-column. Chemical Engineering Science, v. 40, n. 3, p. 449-462, 1985.

KITSCHA, J.; KOCAMUSTAFAOGULLARI, G. Breakup criteria for fluid particles. International Journal of Multiphase Flow, v. 15, n. 4, p. 573-588, 1989.

LETZEL, H. M.; SCHOUTEN, J. C.; KRISHNA, R.; VAN DEN BLEEK, C. M. Gas holdup and mass transfer in bubble column reactors operated at elevated pressure. Chemical Engineering Science, v. 54, p. 2237-2246, 1999.

LETZEL, M.; STANKIEWICZ, A. Gas hold-up and mass transfer in gas-lift reactors operated at elevated pressure. Chemical Engineering Science, v. 54, p. 5153-5157, 1999.

LINEK, V.; BENES, P.; VACEK, V. Dynamic pressure method for kLa measurement in large-scale bioreactors. Biotechonology and Bioengineering, v. 33, p. 1406-1412, 1989.

MARQUARDT, D. W. An algorithm for least-squares estimation of nonlinear parameters. Journal of the Society for Industrial and Applied Mathematics, v. 11, n. 2, p. 431-441, 1963.

ONKEN, U.; WEILAND, P. Airlift fermenters: construction, behavior and uses. Advanced Biotechnology Processes, p. 67-95, 1983.

STUDIER, F.W. Protein production by auto-induction in high-density shaking culture. Protein Expression and Purification, v.41, p. 313-322, 2005.

TALAIA, M. A. R. Terminal velocity of a bubble rise in a liquid column. World Academy of Science Engineering and Technology, v. 22, p. 264-268, 2008.

WILKINSON, P. M.; DIERENDONCK, L. L. V. Pressure and gas density effects on bubble break-up and gas hold-up in bubble columns. Chemical Engineering Science, v. 45, n. 8, p. 2309-2315, 1990. 